Planta (Berl.) 94, 253-264 (1970)

(C) by Springer-Verlag 1970

\title{
Conversion of Furfural to Furoic Acid and Furfuryl Alcohol by Neurospora Ascospores
}

\author{
Frederick I. Emiers \\ Department of Botany and Bacteriology, University of South Florida ] \\ Alfred S. Sussman \\ Department of Botany, University of Michigan
}

Received June 10, 1970

\begin{abstract}
Summary. The metabolism of furfural was studied with regard to possible mechanisms by which the chemical induces germination in ascospores. Incubation of ascospores in furfural resulted in the uptake of a small percent of the furfural, and the conversion of the bulk of it to furoic acid which was in turn converted to furfuryl alcohol. Conversion also occurred in Neurospora mycelium and conidia with the order being furfural to furfuryl alcohol to furoic acid. Conversion appears to be a noninducible enzymatic process localized on the outer surface of the cell. Conversion was completely inhibited without preventing germination indicating that conversion is not involved in the breaking of dormancy in Neurospora ascospores.
\end{abstract}

\section{Introduction}

Emerson (1948) demonstrated that in addition to heat shock (Shear et al., 1927) furfural would also cause the activation or the breaking of dormancy in Neurospora ascospores. This method of breaking dormancy was similar to heat shock in that the response of the spores to the chemical was rapid, high percentages of the spores germinated, and there was an optimal concentration for the activator. Furfural activation differed from heat activation in that it was not as reversible as heat activation, the response of the activated spores to various poisons differed, and the ascospores showed an optimal time for furfural sensitivity (a period of ripeness) which was not shown for heat. With regard to the period of ripeness, it has been demonstrated by Sussman (1954) that a nonactivating heat pre-treatment of $46^{\circ} \mathrm{C}$ for 30 minutes, causes ascospores of any age to respond maximally to furfural.

There have been several studies on the mechanism of heat activation but few on the mechanism of furfural activation. Consequently, it was important to study furfural metabolism by ascospores with particular regard to its role in spore activation.

17 Planta (Berl.), Bd. 94 


\section{Materials and Methods}

\section{Preparation of Ascospores}

Ascospores of Neurospora tetrasperma were grown and harvested by technique, described previously (Goddard, 1935) except that the EDTA treatment was lenthened from 24 hours to 7 days.

The ascospores are designated according to their treatment as follows:

Dormant spores are those which have not received any treatment beyond the initial EDTA wash. Heat-sensitized spores are dormant spores which have received a subactivational heat-treatment (Sussman, 1954) which causes the spores to respond maximally to furfural. Furfural-activated ascospores are those which have been heat-sensitized and then immediately incubated in 1 to $4 \times 10^{-3} \mathrm{M}$ furfural, which is the optimal concentration range for spore activation. Unless otherwise mentioned, these spores were continuously incubated in furfural. Furfural activated ascospores germinate some 3 hours after the addition of furfural. In some experiments, heat-activated ascospores were used. These spores were heated at $60^{\circ} \mathrm{C}$ for 10 minutes. Like furfural-activated spores, they germinated some 3 hours after being returned to room temperature.

\section{Preparation of Conidiospores}

Conidia of $N$. crassa strain 89601 A were grown on solid sucrose minimal media (Ryan, 1950). Just prior to use, they were removed from the mycelium by shaking with water and separated from mycelial fragments by filtration through glass wool.

\section{Preparation of Mycelium}

In some experiments, mycelium of $N$. crassa $89601 \mathrm{~A}$ and $N$. tetrasperma 374.4 were tested for conversional activity. Mycelium was grown in $500 \mathrm{ml}$ of liquid sucrose minimal media in a $2,800 \mathrm{ml}$ fernback flask. A heavy innoculum was used such that 24 hours later the mycelial fragments were still small enough to be pipetted. The mycelium was washed with distilled water prior to use.

\section{Assay of Furfural, Furoic Acid and Furfuryl Alcohol}

Furfural concentration was estimated by either radioactivity or U.V. $(277 \mathrm{~nm})$ absorption measurements of the incubation media after removal of the spores by filtration. In later experiments, aliquots of the incubation media were scanned from 300 to $200 \mathrm{~nm}$ with a Beckman DBG recording spectrophotometer. By the appropriate use of standard curves using authentic compounds (Aldrich Chemical Co.), it was possible to determine the concentration of furfural, furoic acid, and furfuryl alcohol in each sample. In most of the experiments the basic media contained only distilled water and furfural or related compounds to avoid heavy U.V. absorption of salts around 220 to $200 \mathrm{~nm}$.

\section{Results}

The incubation of Neurospora ascospores in $\mathrm{C}^{\mathbf{1 4}}$-furfural yielded different estimates of the furfural concentration remaining in the medium depending on the method of measurement. As can be seen in Fig. 1, U.V. absorption measurements show a steadily decreasing furfural con- 


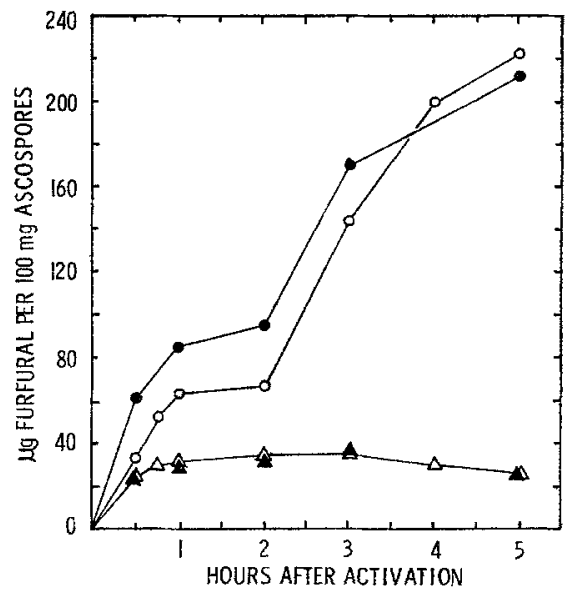

Fig. 1. Furfural uptake and conversion in normal ascospores as determined by radioactivity (filled triangle) and U.V. absorption (filled circles) measurements. The open circles and open triangles represent conversion and uptake respectively for ascospores washed in $0.1 \mathrm{~N} \mathrm{HCl}$ for 30 minutes to kill contaminants before being ineubated in $10^{-3} \mathrm{M}$ furfural

Table 1. Analysis of the activation medium after 7 hours of ascospore incubation

\begin{tabular}{lrr}
\hline Fraction & $\begin{array}{c}\text { CPM } \\
\text { average }\end{array}$ & $\begin{array}{c}\text { Per cent } \\
\text { average }\end{array}$ \\
\hline Furfural & 9,931 & 9.0 \\
Neutral & 74,703 & 68.5 \\
Acid & 7,680 & 7.0 \\
Average recovery & 92,314 & 84.5 \\
\hline
\end{tabular}

centration while radioactivity measurements show an initial decrease after which the concentration remains stable. While these results appear to conflict, they may be correlated by assuming that each method monitors a different response of the ascospores to furfural. The U.V. measurements could indicate the disappearance of furfural as furfural while the radioactivity measurements could indicate the disappearance of furfural or byproducts from the medium.

To test this possibility, the incubation media was analyzed using several methods. Table 1 represents the findings of an ion exchange column separation of the medium after 7 hours of ascospore incubation. The medium was separated into three fractions: furfural, neutral, and 
acidic. As shown, a small amount of material as gauged by radioactivity resided in the furfural and acid fractions while the majority of activity was found in the neutral fraction.

A U.V. absorption spectrum was run on the peak of each fraction and only the neutral fraction showed a distinct peak at $217 \mathrm{~nm}$ which is the absorption peak for furfuryl alcohol.

In a second experiment germinated ascospores were incubated in concentrated furfural for 8 hours, after which the medium was analyzed by gas chromatography for furfural and furfuryl alcohol. The sample was separated using a 6 foot carbo wax 600 column with hydrogen as the eluting gas. The retension times of the volatile components found in the medium were identical to those of authentic furfural and furfuryl alcohol either separately or in a mixture. With the exception of water, no other peaks were found indicating no other volatile compound in the medium.

Furoic acid was also identified as a component in the media by comparison with authentic furoic acid in the paper chromatography system of Buch et al. (1952).

The ascospore activation medium was also examined by comparing the U.V. absorption spectra to spectra of authentic samples of furfural, furoic acid and furfuryl alcohol. Fig. 2 shows the results obtained for ascospores by this type of analysis. The peaks found correspond exactly to those for the authentic compounds. Also, other than these three, no additional peaks were found.

Further work also demonstrated the uptake of furfural and is discussed in detail elsewhere. Consequently, it can be concluded that two processes occur when ascospores are incubated in furfural: furfural conversion and uptake. Conversion can only be measured by U.V. absorption and under most circumstances, uptake is measured only by radioactivity measurements.

The finding of furoic acid and furfuryl alcohol as conversion products of furfural has been reported by Searles and French (1964). In their work it was found that the uredospores of Puccinia produced the alcohol while contaminant bacteria were responsible for acid production.

To avoid the problem of contamination, ascospores were shaken in 0.1 N HCl for 30 minutes to kill contaminants before being washed free of the acid. Acid treatment has no adverse effect on the germination of ascospores. These spores were then heat-sensitized and incubated in furfural as before. The results obtained by radioactivity measurements (uptake) were identical to those for non acid-treated spores (Fig. 1).

The U.V. absorption measurements (conversion) showed lag of about $\mathbf{5 0} \%$ during the first 120 minutes after furfural addition. However, after this lag, the conversional activity is similar to that of non-acid treated 


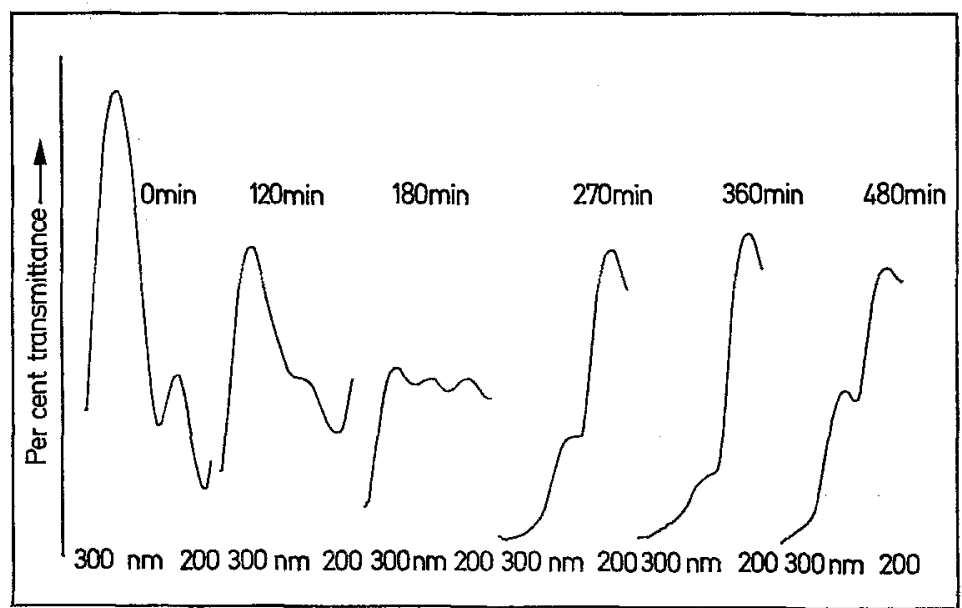

Fig. 2. Furfural conversion in ascospores monitored by U.V. spectral scans from $300 \mathrm{~nm}$ to $200 \mathrm{~nm}$. In the " 0 " minute sample a standard furfural peak is seen with peaks at 277 and $227 \mathrm{~nm}$. In the 180 minute sample, 3 peaks are seen corresponding to furfural (277), furoic acid (245) and furfuryl alcohol (217) absorption maxima.

In the $\mathbf{3 6 0}$ minute sample a sharp peak for furfuryl alcohol is seen

spores (Fig. 1). The initial lag is not thought to be due to contaminants but rather to the effect of the acid on the enzyme(s) responsible for conversion. Thus, the increased activity after germination (approx. 3 hours) is probably due to new enzyme molecules unaffected by the acid.

In many other experiments, direct plate counts of bacterial and fungal contaminants were always found to be extremely low. Also, the results were far too consistent to be due to contaminants.

\section{Conversion Order}

Ascospores. The sequence of appearance of conversion products as well as their concentration in ascospores is shown in Fig. 2. At " 0 " time a standard furfural spectrum is seen with a major peak at $277 \mathrm{~nm}$ and a minor peak at $227 \mathrm{~nm}$. As incubation increases the major furfural peak decreases and a shoulder appears at approximately $245 \mathrm{~nm}$ which is the single absorption peak for furoic acid. At 180 minutes (the time of germination) three peaks can be seen at 277,245 , and $217 \mathrm{~nm}$. This last peak corresponds to the single absorption peak for furfuryl alcohol. At 270 and 300 minutes the furfuryl alcohol peak increases while the furoic acid peak decreases. At these times, no furfural peak is seen. If the experiment is continued, as the hyphae develop (480 minutes) furfuryl alcohol is converted back to furoic acid. Thus for ascospores, the conversional order is furfural-furoic acid-furfuryl alcohol-furoic acid. 


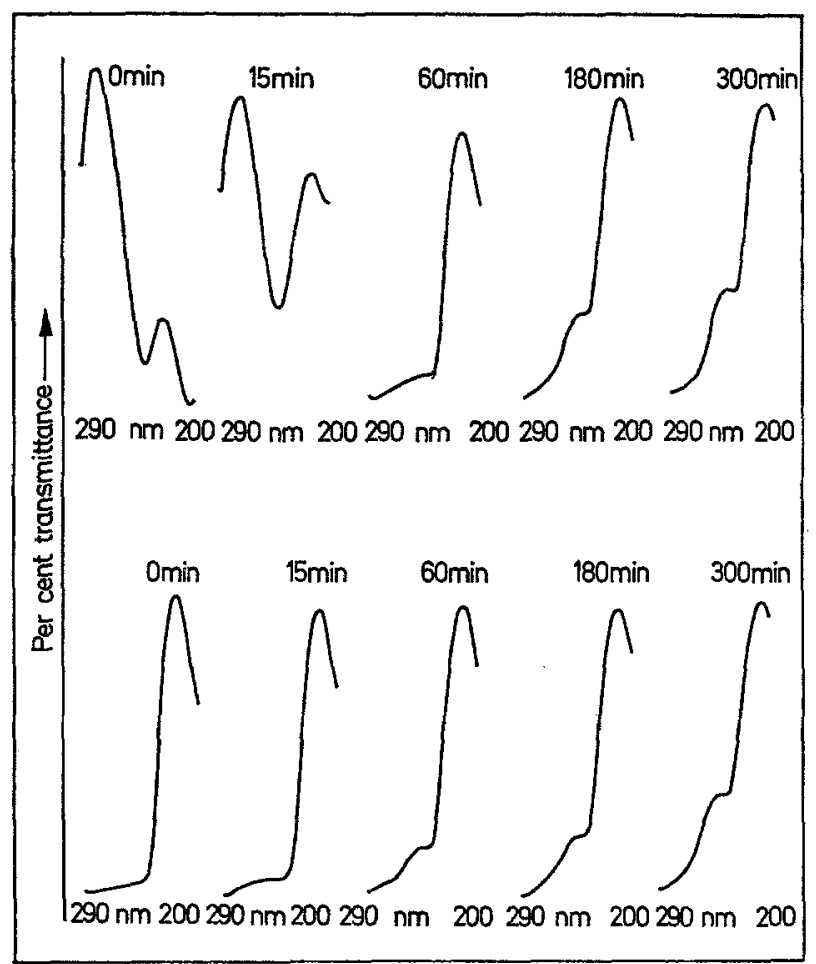

Fig. 3. Conversion of furfural (upper series) and furfuryl alcohol (lower series) by mycelium. The concentration of mycelium was $12.4 \mathrm{mg}$ (dry wt.) for the furfural series and $16.6 \mathrm{mg}$ (dry wt.) for the furfuryl alcohol series while the concentration of both chemicals was $4 \times 10^{-3} \mathrm{M}$

In this sequence apparently the furfural to furoic acid step is carried out by activated but non-germinated spores, the furoic acid to furfuryl alcohol step by germinating spores, and the furfuryl alcohol back to furoic acid step is carried out by the developing vegetative mycelium. This correlation of conversion activity with morphological change was tested by incubating heat-sensitized ascospores in furoic acid $\left(4 \times 10^{-3} \mathrm{M}\right)$ which at all concentrations is ineffective in activating spores. In this experiment, furoic acid was not converted to furfuryl alcohol nor did germination occur. Also, when heat-activated ascospores, and ascospores incubated briefly in furfural to activate them after which the excess furfural was removed by washing, were incubated in furoic acid, conversion in both groups did not occur until the spores germinated. 


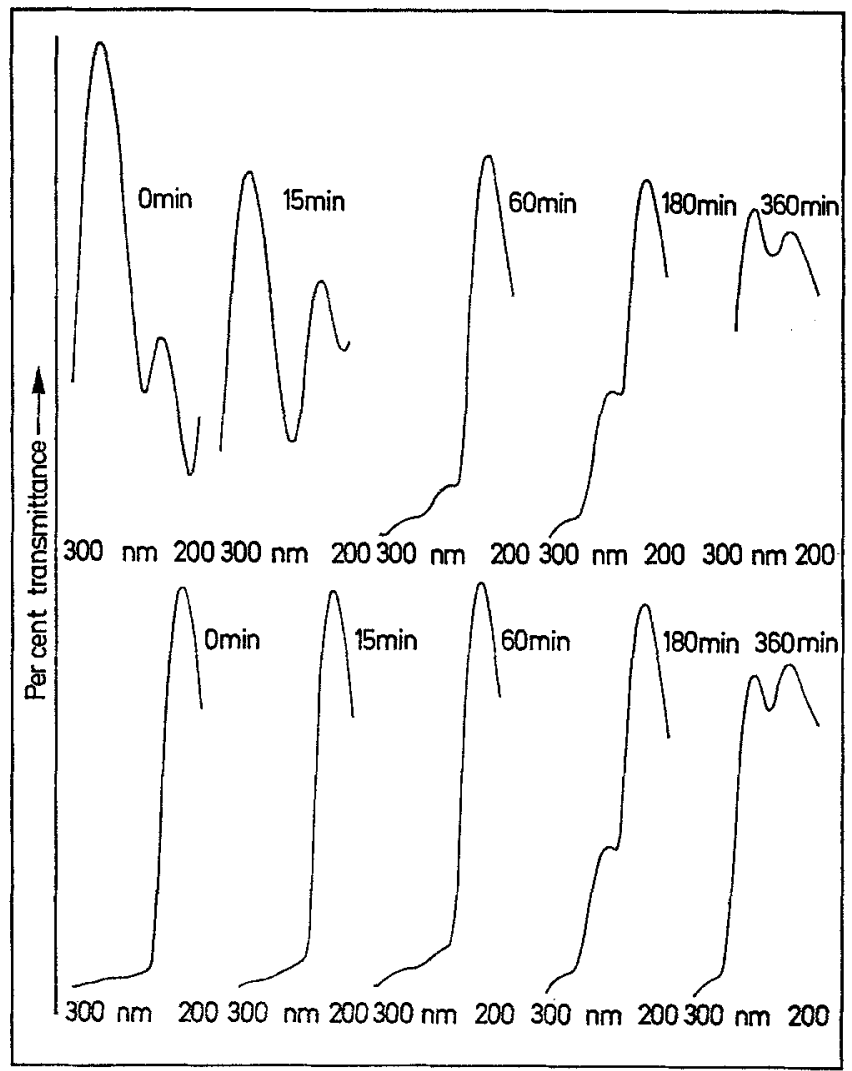

Fig. 4. Conversion of furfural (upper series) and furfuryl alcohol (lower series) by $N$. crassa conidia. Conidial concentration was $4 \mathrm{mg}$ (dry wt.) per $\mathrm{ml}$ and the furfural and furfuryl alcohol concentrations were each $4 \times 10^{-3} \mathrm{MI}$

The suggestion that the conversion of furfuryl alcohol to furoic acid is due to the insuing vegetative growth was examined by using mycelium and conidia.

\section{Mycelium and Conidia}

The results of incubating $N$. crassa mycelium in furfural and furfuryl alcohol are shown in Fig. 3. As can be seen, furfural conversion occurs, and the order is now furfural to furfuryl alcohol to furoic acid. The furfuryl alcohol to furoic acid step substantiates the previous supposition for the ascospores.

Also, the appearance of acid occurs at the same time whether the mycelium is incubated in furfural or furfuryl alcohol indicating that the 


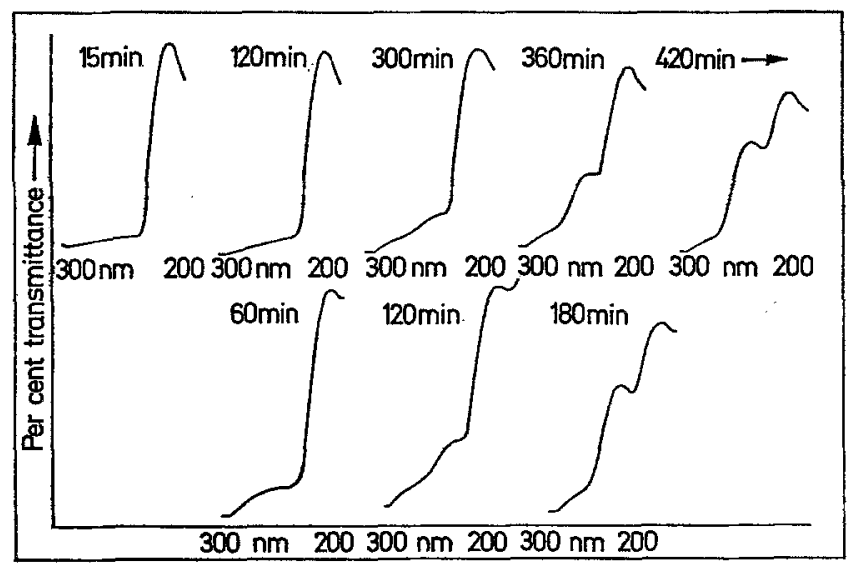

Fig. 5. Conversion of furfuryl alcohol by furfural-activated ascospores (upper series) and heat-activated ascospores (lower series). The 60 minute sample in the heatactivated series represents 300 minutes of incubation in distilled water after heat shock and then 60 minutes in the presence of furfuryl alcohol. In terms of time after activation this sample is equal to the 360 minute furfural-activated sample

reaction leading to furfuryl alcohol is more active than that leading to furoic acid.

As might be expected, $N$. tetrasperma mycelium gave the same results as $N$. crassa and consequently is not shown.

The incubation of $N$. crassa conidia in furfural and furfuryl alcohol is essentially similar to those for mycelia and is shown in Fig. 4.

Thus, furfural conversion producing both furoic acid and furfuryl alcohol occurs in the ascospores, mycelium, and conidia. In addition, all of the enzymes responsible appear to be constitutive enzymes. This was further evidenced by a comparison between heat-activated and furfuralactivated spores. The heat-activated group was incubated in distilled water and had no contact with furfural of furfuryl alcohol. The course of conversion was monitored at regular intervals and at 300 minutes when the furfural activated group showed a small accumulation of furoic acid, furfuryl alcohol was added to the heat-activated spores. As can be seen in Fig. 5, the conversion of alcohol to acid occurs in the heatactivated group without a lag. If the rates of acid appearances are plotted, the slope of the lines are identical in both groups of spores.

\section{Stoichiometry of Conversion}

To ascertain whether conversion occurred as seen in the spectral analysis, a balance sheet was made for each sample in the conversion sequence for ascospores and conidia. If in these balance sheets, large 
Table 2. Stoichiometry of the conversion of furfural to furoic acid and furfuryl alcohol. Data based on conversion per $100 \mathrm{mg}$ ascospores

\begin{tabular}{rcccc}
\hline $\begin{array}{l}\text { Time } \\
(\mathrm{min})\end{array}$ & $\begin{array}{l}\text { Furfural } \\
(\mu \mathrm{g})\end{array}$ & $\begin{array}{l}\text { Furoic } \\
\text { acid } \\
(\mu \mathrm{g})\end{array}$ & $\begin{array}{l}\text { Furfuryl } \\
\text { alcohol } \\
(\mu \mathrm{g})\end{array}$ & $\begin{array}{l}\text { Total } \\
(\mu \mathrm{g})\end{array}$ \\
\hline 0 & $\mathbf{3 7 5}$ & 0 & 0 & 375 \\
5 & 274 & $\mathbf{1 7}$ & 0 & 291 \\
15 & 256 & 45 & 0 & 301 \\
30 & 233 & 40 & 0 & 273 \\
60 & 220 & 50 & 0 & $\mathbf{2 7 0}$ \\
120 & 174 & 81 & 0 & 255 \\
180 & 86 & 81 & 115 & 282 \\
270 & 0 & 67 & 203 & 270 \\
300 & 0 & 47 & 274 & 321 \\
360 & 0 & 33 & 297 & 330 \\
\hline
\end{tabular}

Table 3. Stoichiometry of the conversion of furfural and furfuryl alcohol by conidia of $N$. crassa. Data based on conversion per $13.5 \mathrm{mg}$ dry weight of conidia

\begin{tabular}{rrrrr}
\hline $\begin{array}{r}\text { Time } \\
(\min )\end{array}$ & $\begin{array}{l}\text { Furfural } \\
(\mu \mathrm{g})\end{array}$ & $\begin{array}{l}\text { Furoic } \\
\text { acid } \\
(\mu \mathrm{g})\end{array}$ & $\begin{array}{l}\text { Furfuryl } \\
\text { alcohol } \\
(\mu \mathrm{g})\end{array}$ & $\begin{array}{l}\text { Total } \\
(\mu \mathrm{g})\end{array}$ \\
\hline 0 & 448 & 0 & 0 & $\mathbf{4 4 8}$ \\
5 & $\mathbf{3 7 0}$ & 0 & 61 & $\mathbf{4 3 1}$ \\
15 & 235 & 0 & 226 & $\mathbf{4 6 1}$ \\
$\mathbf{3 0}$ & 110 & 0 & $\mathbf{3 5 0}$ & $\mathbf{4 6 0}$ \\
60 & 0 & 19 & 441 & 460 \\
120 & 0 & 29 & 432 & $\mathbf{4 6 1}$ \\
$\mathbf{1 8 0}$ & 0 & 89 & $\mathbf{3 8 5}$ & $\mathbf{4 7 4}$ \\
$\mathbf{2 4 0}$ & 0 & 163 & 274 & $\mathbf{4 3 7}$ \\
$\mathbf{3 0 0}$ & 0 & $\mathbf{2 3 5}$ & 228 & $\mathbf{4 6 3}$ \\
$\mathbf{3 6 0}$ & 0 & 296 & $\mathbf{1 6 8}$ & $\mathbf{4 6 4}$ \\
0 & 0 & 0 & $\mathbf{5 3 8}$ & $\mathbf{5 3 8}$ \\
5 & 0 & 12 & $\mathbf{5 1 0}$ & $\mathbf{5 2 2}$ \\
15 & 0 & $\mathbf{1 2}$ & $\mathbf{5 1 3}$ & $\mathbf{5 2 5}$ \\
30 & 0 & 12 & $\mathbf{5 1 4}$ & 526 \\
60 & 0 & 12 & $\mathbf{5 1 3}$ & $\mathbf{5 2 5}$ \\
120 & 0 & $\mathbf{3 7}$ & 490 & $\mathbf{5 2 7}$ \\
180 & 0 & 90 & $\mathbf{4 5 1}$ & 541 \\
$\mathbf{2 4 0}$ & 0 & 173 & $\mathbf{3 6 4}$ & $\mathbf{5 3 7}$ \\
300 & 0 & $\mathbf{2 4 6}$ & 297 & $\mathbf{5 4 3}$ \\
$\mathbf{3 6 0}$ & 0 & 291 & $\mathbf{2 3 0}$ & $\mathbf{5 2 1}$ \\
\hline
\end{tabular}

amounts of material cannot be accounted for, other compounds which do not appear in the U.V. spectra may occur. These balance sheets are seen in Tables 2 and 3 . As can be seen after the initial decrease in ascospores 


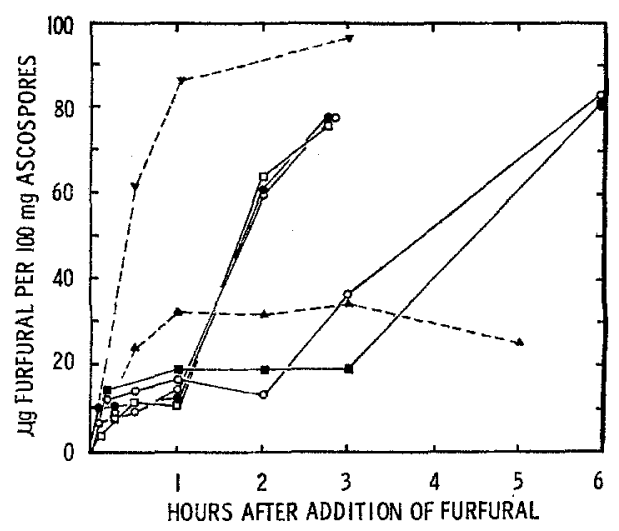

Fig. 6. Uptake and conversion of furfural by ascospores treated with Clorox for 2 minutes (filled circles), 5 minutes (open circles), 10 minutes (open squares), 30 minutes (hexagons), and 60 minutes (filled squares) before incubation in $2 \times$ $10^{-3} \mathrm{M}$ furfural. All measurements made by U.V. absorption. For comparison, furfural uptake (triangles) and conversion (inverted triangles) from a previous experiment using $\mathrm{C}^{14}$-furfural with untreated ascospores are indicated

due to uptake, the material remaining as conversion proceeds is essentially $100 \%$ indicating no other compounds are involved. Conidia did not show an initial decrease indicating that little if any uptake of the compounds occurs. The balance sheets for conidia also indicated that no other compounds are involved.

\section{The Effect of Various Surface Agents on Conversion}

In attempt to determine the relationship of conversion to activation, ascospores were pretreated with various compounds which might affect processes occurring in the spore surface. Since most of the furfural and conversion products remain in the medium, it is likely these reactions occur outside any permeable barriers of the cell.

Pretreatment of ascospores for as long as 24 hours with pepsin, trypsin, protease, peptidase, sodium laryl sulfate and sodium deoxycholate had no detectable effet on conversion.

Clorox, however, did inhibit conversion markedly (Fig. 6). Ascospores were soaked in $20 \%$ standard Clorox solution to remove the outer spore walls (Lowry et al., 1958) and then washed free of the excess Clorox and activated in furfural. Although complete wall removal from all the spores was not achieved, all of the remaining spore walls were swollen. Furfural concentration was measured at various times by U.V. absorption. While such measurements normally only indicate conversion, it is felt that the U.V. measurements for the first $60-120$ minutes after furfural addition represent primarily uptake, showing a $50 \%$ reduction in uptake and a 
severe, if not total, inhibition of conversion. After 60-180 minutes, depending on the time of Clorox incubation, these measurements are felt to represent primarily conversion.

Although the viability of these spores was decreased by treatment, spore germination did occur ranging from $14 \%$ for the 60 minute Clorox sample to $46 \%$ for the 5 minute sample.

In subsequent experiments in which $5 \%$ and $1 \%$ Clorox solutions were used for 5 and 10 minutes, similar results were obtained showing an inhibition in conversion for 60-120 minutes. However, in these spores germination was improved, reaching an average of $80 \%$ for the $1 \%$ solution.

\section{Conversion in Ascospore Extracts}

Conversional activity was tested in cell free extracts using both fractionated and whole spore extracts. In none of these extracts was it possible to detect any evidence of conversion.

\section{Discussion}

There are two aspects of conversion which warrant consideration: conversion itself as a series of reactions occurring in Neurospora, and the relationship of conversion to ascospore activation.

With regard to conversion as a specific entity, it appears to be somewhat different in Neurospora, as compared to other fungi. Searles et al. (1964) have reported the reduction of furfural to furfuryl alcohol by the uredospores of Puccinia. They also reported the conversion of cinnamaldehyde to cinnamylalcohol by Puccinia uredospores and conidia of Penicillium spp. They found contaminant bacteria produced the acid of each aldehyde. Morimoto et al. (1967) reported furfuryl alcohol production from furfural by yeast. Furoic acid was also found in the media in very small amounts presumably produced by the yeast. Consequently, aldehyde to alcohol conversion may be common in fungi while aldehyde to acid and alcohol to acid conversion as found in Neurospora may be somewhat restrictive.

Conversion is apparently enzymatic since high temperatures completely inhibit the process and $\mathrm{HCl}$ and Clorox inhibit it to varying degrees. Also, cell-free extracts of ascospores and mycelium preparations obbained by mild grinding have never shown conversion activity. However, extensive heat, inhibitor, or co-factor studies have not been carried out.

From the data at hand, replotting of the absorption spectra shows that the appearance of conversion products are in all cases straight or essentially straight lines. Thus, the enzymes involved apparently do not arise by induction.

The effects of Clorox and acid treatments appear to be confined to the spores exterior since germination is not prevented. This indicates 
that conversion in ascospores at least occurs on the outer surface of the spores.

The significance of conversion in ascospore activation may be inferred from the Clorox experiment and the order of conversion products. Clorox treated spores indicate that conversion can be completely inhibited while still permitting germination.

The first formed product in ascospores is furoic acid which is extremely ineffective in activating the spores. Also, furfuryl alcohol is first produced at the time of germination and is not produced in nongerminating spores from furoic acid. Consequently, conversion products cannot be considered as possible activators when spores are initially incubated in furfural.

Taken together these results indicate that conversion, while it utilizes most of the furfural required to activate ascospores, is not involved in spore activation. Consequently, the furfural taken up by the ascospore must fulfill the role of spore activator.

Part of this work was included in a dissertation submitted to the Rackham Graduate School of the University of Michigan in partial fulfillment of the requirements for the Doctor of Philosophy degree in Botany by Frederick I. Eilers.

The authors wish to thank J. M. Patrick for his technical assistance.

\section{References}

Buch, M. L., Montgomery, R., Porter, W. L.: Identification of organic acids on paper chromatograms. Anal. Chem. 24, 489-491 (1952).

Emerson, M. R.: Chemical activation of ascospore germination in Neurospora crassa. J. Bact. 55, 327-330 (1948).

Goddard, R. R.: The reversible heat-activation inducing germination and increased respiration in the ascospores of Neurospora tetrasperma. J. gen. Physiol. $19,45-60(1935)$.

Lowry, R. J., Sussman, A. S.: Wall structure of ascospores of Neurospora tetrasperma. Amer. J. Bot. 45 (5), $397-403$ (1958).

Morimoto, S., Hirashima, T., Ohashi, M.: Studies on fermentation products from aldehyde by microorganisms. II. The fermentative production of furfuryl alcohol from furfural by yeast (Part II). J. Ferment. Tech. 46 (4), 276-287 (1967).

Ryan, F. T.: Selected methods of Neurospora genetics. Meth. med. Res. 3, 5I-75.

Searles, R. B., French, R. C.: Conversion of furfural and cinnamaldehyde by uredospores of wheat stem rust and associated bacteria. Bot. Gaz. 125, 146-148 (1964).

Shear, C. L., Dodge, B. D.: Life histories and heterothallism of the red bread-mold fungi of the Monilia sitophila group. J. agric Res. 34, 1019-1043 (1927).

Sussman, A. S.: The sensitization of ascospores to chemical activators by heat treatment. Mycologia 46, 143-150 (1954).

Dr. F. I. Eilers

Department of Botany and Bacteriology

University of South Florida

Tampa, Florida 33620, U.S.A. 